\title{
Rapid Foreign Object Detection System on Seaweed Using VNIR Hyperspectral Imaging
}

\author{
Dong-Hoon Kwak ${ }^{1}{ }^{1}$, Guk-Jin Son ${ }^{1}$, Mi-Kyung Park ${ }^{2}{ }^{\circledR}$ and Young-Duk Kim ${ }^{1, *}$ \\ 1 ICT Research Institute, DGIST, Daegu 42988, Korea; gns9452@dgist.ac.kr (D.-H.K.); \\ sudopop@dgist.ac.kr (G.-J.S.) \\ 2 School of Food Science and Biotechnology, Kyungpook National University, Daegu 41566, Korea; \\ parkmik@knu.ac.kr \\ * Correspondence: ydkim@dgist.ac.kr; Tel.: +82-53-785-4641
}

Citation: Kwak, D.-H.; Son, G.-J.; Park, M.-K.; Kim, Y.-D. Rapid Foreign Object Detection System on Seaweed Using VNIR Hyperspectral Imaging. Sensors 2021, 21, 5279. https:// doi.org/10.3390/s21165279

Academic Editor: Vassilis S. Kodogiannis

Received: 27 July 2021

Accepted: 3 August 2021

Published: 4 August 2021

Publisher's Note: MDPI stays neutral with regard to jurisdictional claims in published maps and institutional affiliations.

Copyright: (C) 2021 by the authors Licensee MDPI, Basel, Switzerland. This article is an open access article distributed under the terms and conditions of the Creative Commons Attribution (CC BY) license (https:/ / creativecommons.org/licenses/by/ $4.0 /)$.

\begin{abstract}
The consumption of seaweed is increasing year by year worldwide. Therefore, the foreign object inspection of seaweed is becoming increasingly important. Seaweed is mixed with various materials such as laver and sargassum fusiforme. So it has various colors even in the same seaweed. In addition, the surface is uneven and greasy, causing diffuse reflections frequently. For these reasons, it is difficult to detect foreign objects in seaweed, so the accuracy of conventional foreign object detectors used in real manufacturing sites is less than $80 \%$. Supporting real-time inspection should also be considered when inspecting foreign objects. Since seaweed requires mass production, rapid inspection is essential. However, hyperspectral imaging techniques are generally not suitable for high-speed inspection. In this study, we overcome this limitation by using dimensionality reduction and using simplified operations. For accuracy improvement, the proposed algorithm is carried out in 2 stages. Firstly, the subtraction method is used to clearly distinguish seaweed and conveyor belts, and also detect some relatively easy to detect foreign objects. Secondly, a standardization inspection is performed based on the result of the subtraction method. During this process, the proposed scheme adopts simplified and burdenless calculations such as subtraction, division, and one-by-one matching, which achieves both accuracy and low latency performance. In the experiment to evaluate the performance, 60 normal seaweeds and 60 seaweeds containing foreign objects were used, and the accuracy of the proposed algorithm is $95 \%$. Finally, by implementing the proposed algorithm as a foreign object detection platform, it was confirmed that real-time operation in rapid inspection was possible, and the possibility of deployment in real manufacturing sites was confirmed.
\end{abstract}

Keywords: foreign object detection; hyperspectral imaging; visible and near-infrared; spectroscopy; signal processing; seaweed

\section{Introduction}

Dried seaweed is made by mixing various seaweeds (e.g., laver and sargassum fusiforme) and drying them. It is one of the representative side dishes in South Korea. Seaweed is a well-being food rich in minerals, vitamins, and proteins and low in calories. The Wall Street Journal in the United States reported that it is a Korean superfood [1]. Korean seaweed is an Asian seaweed standard adopted by the Codex Alimentarius Commission. It currently exports to more than 100 countries, with annual exports worth $\$ 600$ million. This is a steadily increasing figure in recent years As it is gaining popularity around the world, hygiene management is also becoming important when manufacturing seaweed.

Since seaweed is mixed with materials and dried during the manufacturing process, there is a high possibility of foreign objects coming out. There are various kinds of foreign objects. Foreign objects such as shrimp shells are harmless even if consumed. However, foreign objects such as screws and stones can damage teeth, and bugs can be at risk of bacterial infection. To prevent this accident, it is very important to detect foreign objects through non-destructive inspection. Seaweed is not easy to detect foreign objects. Because 
it has various colors even in the same seaweed and has uneven and greasy surface. Due to these characteristics, according to factory officials, conventional foreign object detectors currently used in real manufacturing sites have less than $80 \%$ accuracy.

In the food industry, various kinds of foreign object detectors are used. Examples include X-ray detectors, metal detectors, infrared (IR) detectors, and RGB image-based detectors [2-5]. These detectors are slightly different from each other for application purposes. X-ray detectors and metal detectors can only detect foreign objects in certain materials (metal or hard material), and internal inspection is also possible. However, their main drawback is that they cannot detect soft foreign objects such as insects, mold, etc. Meanwhile, the RGB-based detector and IR detector can detect such soft foreign objects by analyzing the color and infrared spectral characteristics of the adsorbed material on the food surface. However, they still have poor performance against foreign objects having a similar color with that of food.

Recently, the detector using hyperspectral imaging is specialized for surface inspection with highly sophisticated spectral analysis that is superior to conventional IR detectors. Hyperspectral imaging technique acquires images in the form of the n-dimensional hypercube. It acquires data not only in the spatial direction but also in the wavelength direction. In addition, these images contain wavelength-specific spectral information, ranging from dozens to hundreds per pixel. Through such advantages, hyperspectral imaging is being actively researched in various fields of the food industry [6-10], such as foreign object detection and quality inspection. Most of the previous works did not explicitly present the real-time processing speed. There are few works that can inspect food on a conveyor belt moving at high speeds in real-time. Moreover, the previous works were to detect only specific foreign objects or inspect food quality in specific situations. However, in the food manufacturing industry, it is necessary to satisfy their production speed and to prepare for unspecified foreign objects. To the best of our knowledge, real-time analysis of seaweed using conveyor belts and hyperspectral imaging is the first study.

In this study, in order to resolve the above-mentioned problem of hyperspectral imaging, we firstly adopt visible and near-infrared (VNIR) hyperspectral imaging techniques [11-13]. The VNIR uses wavelength-specific analysis from visible light ranges $(400-750 \mathrm{~nm})$ to near-infrared ranges $(750-1000 \mathrm{~nm})$. When the camera operates at the full band (224 bands per pixel), full resolution (1024 pixels per line), and full fps (Frames Per Second) (330 fps), it needs to process 229,376 bytes of data per line and $68,812,800$ bytes of data per second. However, it is still difficult to process this vast amount of data in real-time with an accurate inspection. This is the main reason for the absence of a commercialized hyperspectral image-based foreign object detection system, especially in conveyor moving inspection. In order to tackle this problem, the characteristics of the seaweed surface should be considered. In the case of data with non-linear values such as seaweed surface pixels, a deep neural network algorithm (e.g., Convolutional Neural Network (CNN), Region-based Convolutional Neural Network (R-CNN), You Only Look Once (YOLO), etc.) [14-17] can be used for classification and detection. Detection algorithms such as R-CNN and YOLO are frequently used for foreign object detection, but it is difficult to process the spectral dimension of hyperspectral images in a real-time operation. There are various methods for detecting foreign objects in hyperspectral images using CNN, including pixel-wise classification using 1D CNN, 2D CNN through dimensionality reduction, and hypercube classification using 3D CNN [18,19]. These methods allow real-time processing when conveyor belts move at low speeds, but cannot process real-time inspections at the speed required by recent commercial seaweed manufacturers. Commercial seaweed manufacturers require more rapid inspection with a conveyor belt speed of at least $30 \mathrm{~cm} / \mathrm{s}$, which can inspect one piece of seaweed per second. Thus, in order to detect a foreign object of about $1 \mathrm{~mm}$ width, it should be inspected in the environment at least $300 \mathrm{fps}$ because when the conveyor belt moves $30 \mathrm{~cm} / \mathrm{s}$, the camera needs to scan 300 lines/s to have a horizontal resolution of $1 \mathrm{~mm}$. The proposed algorithm focuses on real-time inspections of conveyor belt speed required by recent commercial seaweed manufacturers with accuracy inspection. 
The rest of this paper is categorized into four chapters. Section 2 explains the detailed operation and test methods of the proposed algorithm, Section 3 explains the performance verifications for securing the accuracy and real-time. Finally, concluding remarks with a summary is given in Section 4 .

\section{Materials and Methods}

\subsection{Sample Preparation}

A total of 120 sheets of seaweed were used as samples. These include 60 samples of commercial products and 60 samples of seaweed obtained from the real manufacturing site. The size of seaweed is classified into two types according to its shape. The rectangularshaped seaweed has a length of about $26 \mathrm{~cm}$, and the square-shaped seaweed has a length of about $20 \mathrm{~cm}$. Both types of seaweeds are about $20 \mathrm{~cm}$ wide. Among the seaweeds obtained at the real manufacturing sites, 30 samples are containing foreign objects in the manufacturing process. For commercial products, 30 samples of seaweeds are mixed with foreign objects (e.g., insect, shrimp shell, thread, feather, plastic bag, etc.) mainly generated in the actual field to reproduce seaweeds with foreign objects. Finally, 60 samples of normal seaweed and 60 samples of seaweed containing foreign objects were used in the experiment. In Figure 1, two types of seaweed and foreign substances can be seen.

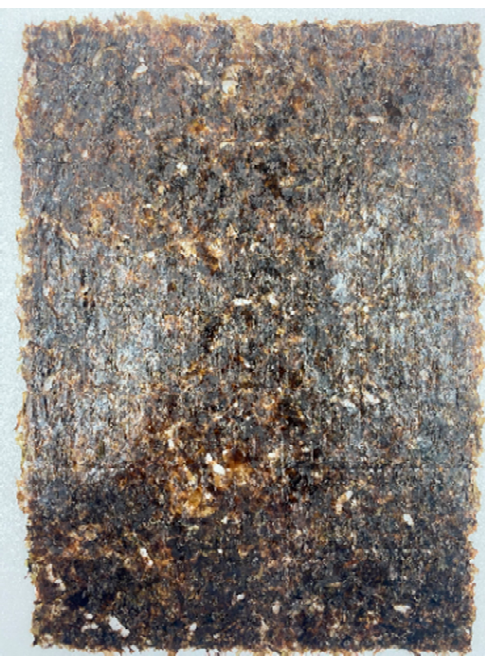

(a)

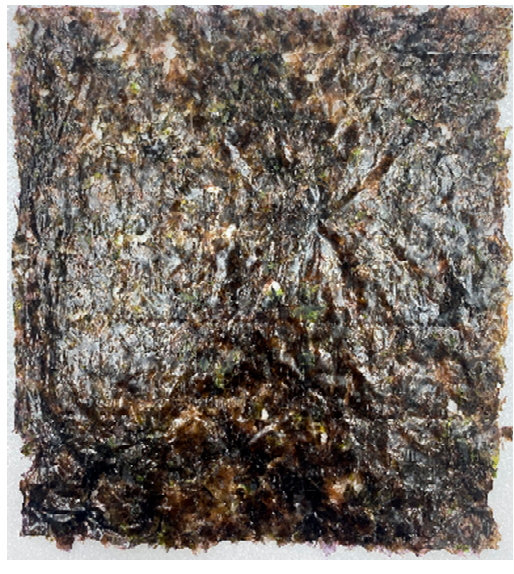

(b)

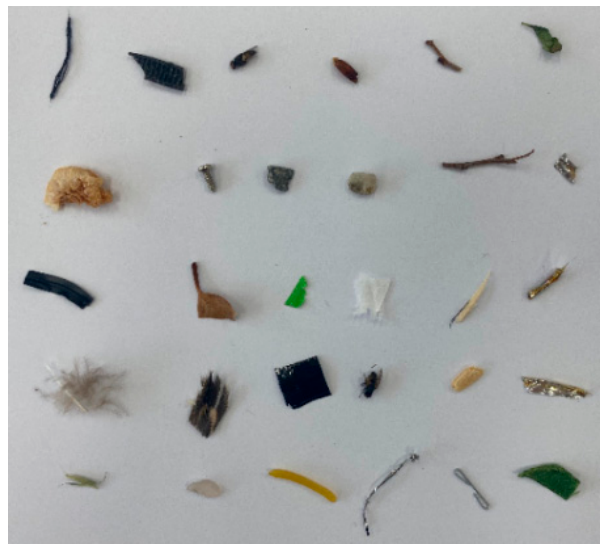

(c)

Figure 1. Seaweed used in the experiment. (a) Obtained from the real manufacturing site (Rectangular-shaped), (b) Commercial products (square-shaped) (c) Examples of foreign objects.

\subsection{Equipment}

The specifications of the push-broom based VNIR hyperspectral camera (FX-10e, Specim, Inc., Oulu, Finland) [20] used in the experiment can be checked in Table 1.

Table 1. Specifications of the FX-10e.

\begin{tabular}{cc}
\hline Parameters & Values \\
\hline Spectral range & $400-1000 \mathrm{~nm}$ \\
Spectral bands & 224 bands (Maximum) \\
Spatial resolution & 1024 pixel \\
Spectral resolution & $2.7 \mathrm{~nm}$ \\
Exposure time & $3.22 \mathrm{~ms}$ \\
Frame rate (Line rate) & $330 \mathrm{fps}$ (Maximum) \\
\hline
\end{tabular}

As a light source, three bulb halogen lamp with a wavelength range of 400 to $1000 \mathrm{~nm}$ was used. When the light source was not used, the characteristics of the sample hardly 
appeared, and when the 1-channel light source was used, it was affected by noise. When a 2-channel light source was used, it was rarely affected by noise, and the characteristics of the sample were well represented. Table 2 shows the light intensity and acquired spectral characteristics of seaweed by the number of channels of the light source.

Table 2. Light intensity and spectral characteristics by number of light source channels.

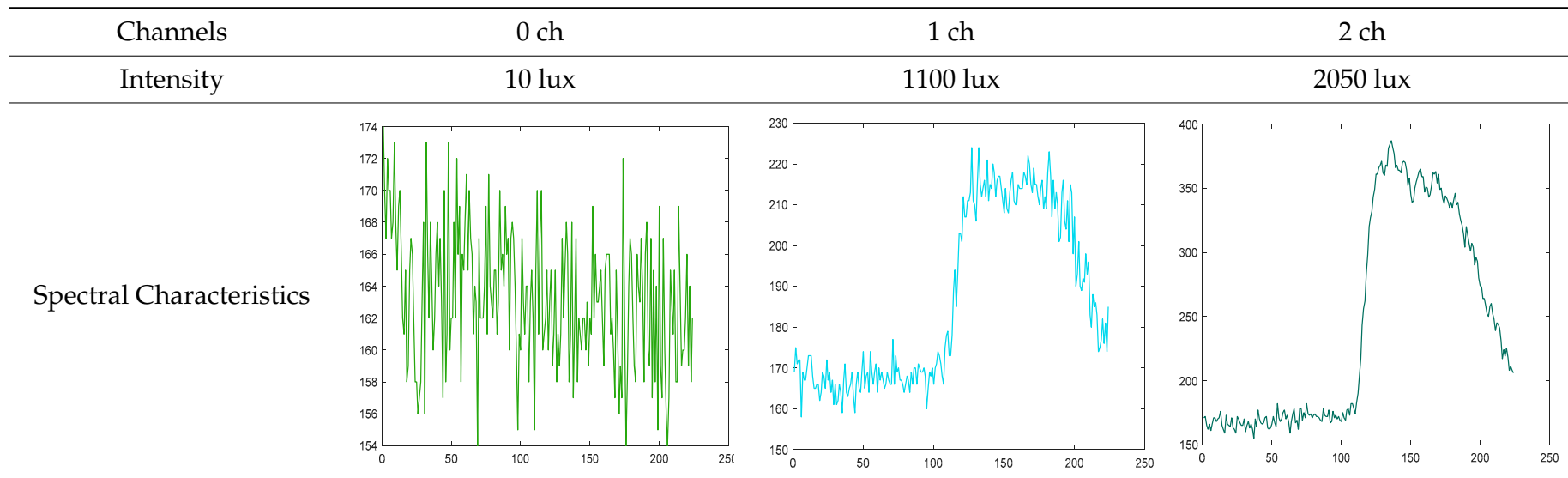

The hyperspectral imaging system used in the experiment can be seen in Figure 2 The $X$-axis of the hyperspectral camera is fixed at the center and can be adjusted with the $Y$-axis. The light source used 2 channels, the angle is adjustable, and it can be moved along the $Y$-axis. The optimal environment can be established by adjusting the light source and camera. The interior of the dark chamber is light-blocked, allowing the same environment to be maintained at all times. The conveyor belt is the same color used in the industry and is driven at $30 \mathrm{~cm} / \mathrm{s}$, which is the same as the speed of the real manufacturing site.

\section{Pushbroom based VNIR Hyperspectral Camera}
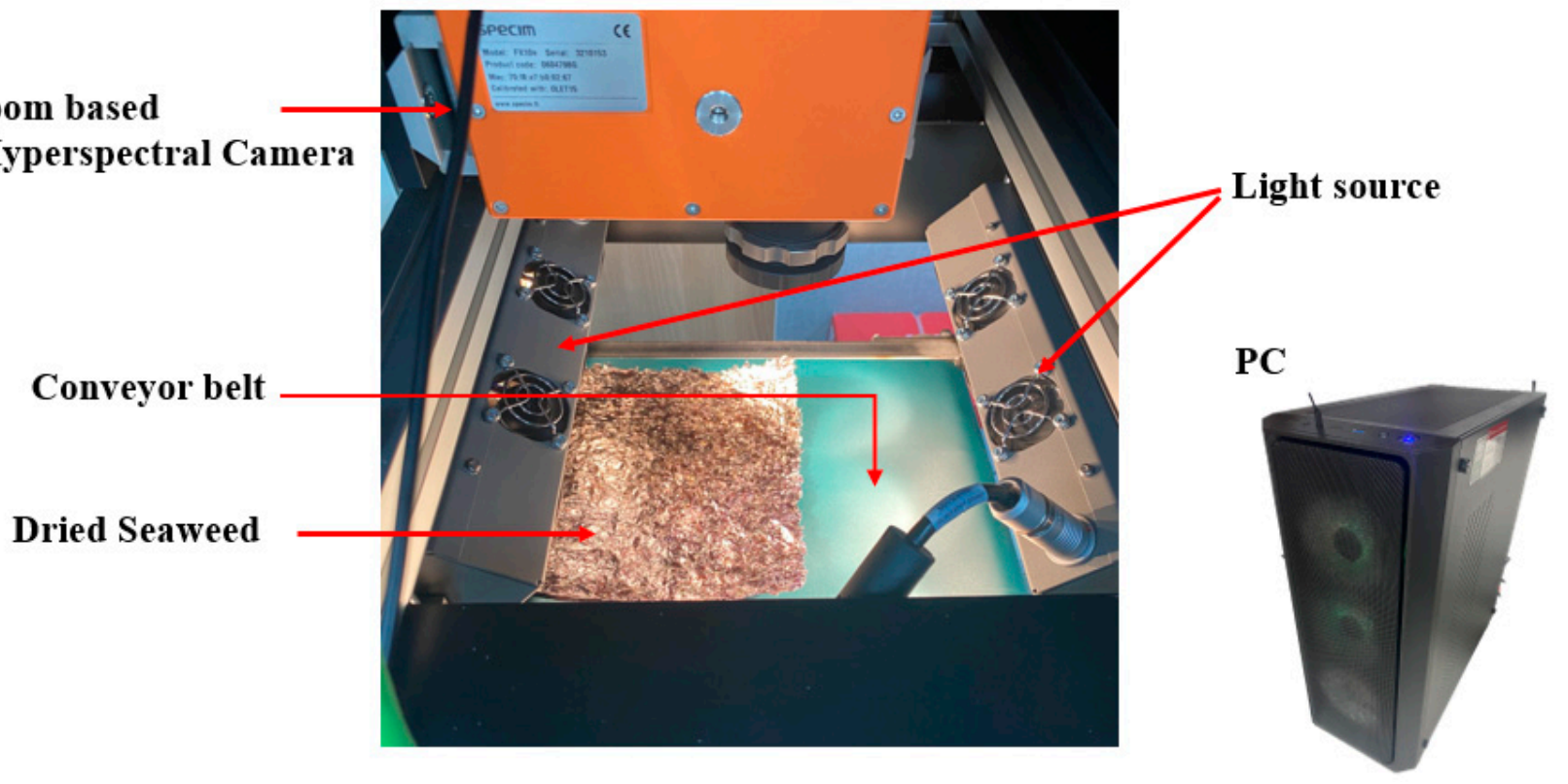

Figure 2. The VNIR hyperspectral imaging system used in the experiment.

\subsection{Hyperspectral Characteristics}

Seaweed is a mixture of various materials, so it has a slightly different various color distribution. In addition, seaweed has an uneven and greasy surface. For these reasons, different spectral characteristics can be obtained even when the same amount of light is irradiated. Figure 3 shows the raw spectral characteristics of 10,000 pixels obtained from 
seaweed and conveyor belt. (The DN of the $Y$-axis is a digital number, which means the intensity of the spectral value.) The spectral characteristics of representative seaweed are shown in Table 2. However, the spectral characteristics of seaweed change due to the color difference and diffuse reflection. When diffuse reflection occurs, the DN value of the wavelength of 500-680 nm increases, and the maximum DN value increases. Therefore, despite the spectral characteristics of pixels obtained from the same seaweed, the offsets between pixels are large. In addition, the spectral characteristics of seaweeds with low DN value distributions have similar spectral characteristics to those of a conveyor belt. To solve this problem, proper calibration is required.

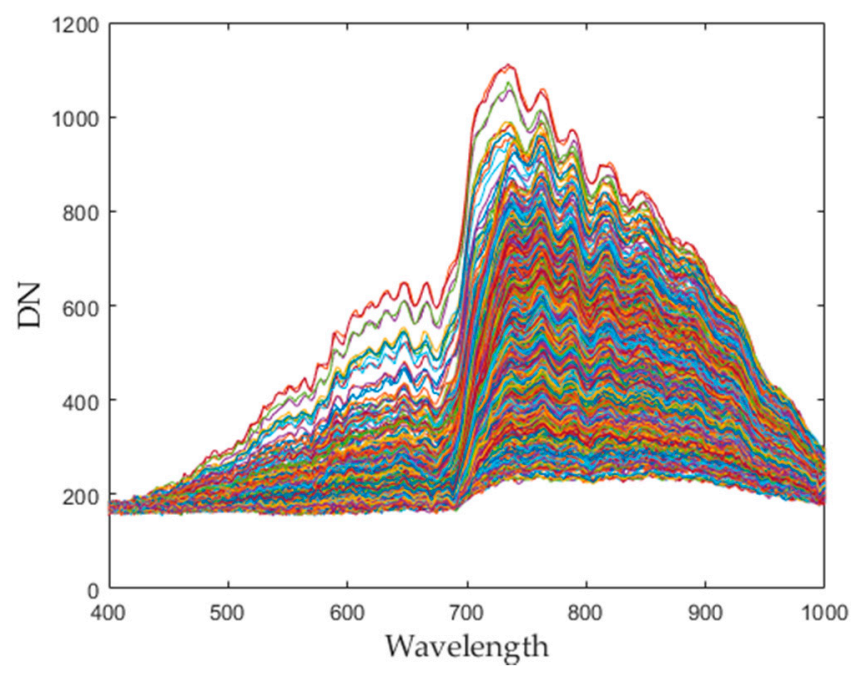

(a)

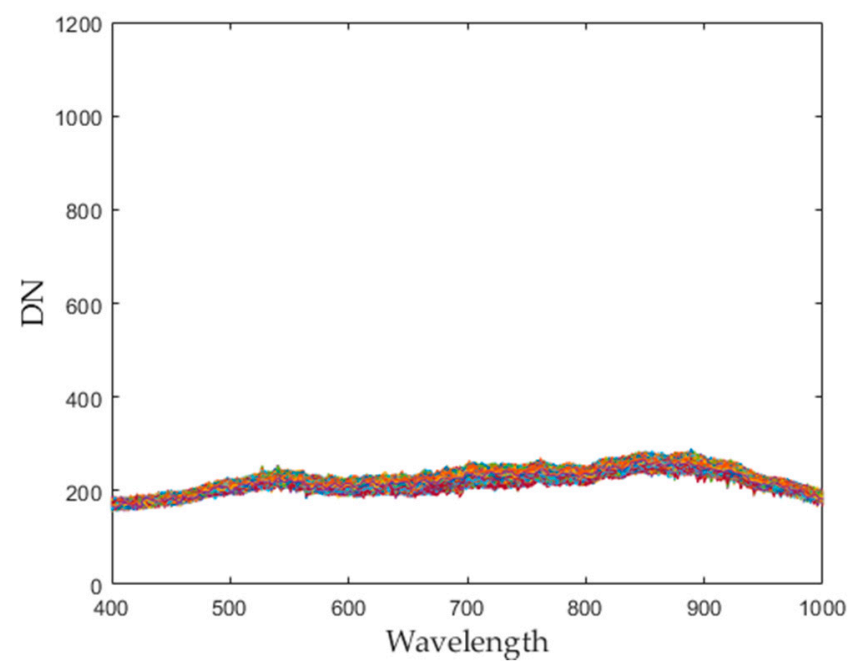

(b)

Figure 3. Spectral characteristics of 10,000 pixels (a) seaweed, (b) conveyor belt.

\subsubsection{Hyperspectral Image Calibration}

In this study, three methods were tested to find the optimal calibration method [21]. Before proceeding with the test, the white-reference value used in the test was obtained through the white balance color checker of $x$-rite Inc. [22], and the dark current value (D) was obtained by covering the camera cover to block the light. The dark current value was used to remove the noise present in the camera. Table 3 shows the results of the three calibration methods. The spectral characteristics used in the three methods were dark current-removed. (1) Per-Norm [23] is a method of normalizing the spectral characteristics in percentage. Per-Norm applied value $\left(P_{n}\right)$ can be obtained by removing the minimum value respectively when dividing the spectral value by the maximum value $\left(r_{\max }\right)$.

(2) Max-Norm is a method of dividing the spectral values by the maximum value. Finally,

(3) Reflection-Norm is a method of dividing the spectral values by the white reference $\left(W_{r e f}\right)$. In general, when processing hyperspectral images, the Reflection-Norm method is popularly used because the effect of the light source is minimized, and the spectral characteristics of the sample can be viewed more accurately. In this case, the Per-Norm method is more effective. The offset between pixels was significantly reduced while maintaining the spectral characteristics of seaweed. Furthermore, since the seaweed and conveyor belt are clearly distinguished, Per-Norm was used as a calibration method. On the other hand, Reflection-Norm cannot be used because even seaweed and conveyor belt pixels are not clearly distinguished. 
Table 3. Calibration results.

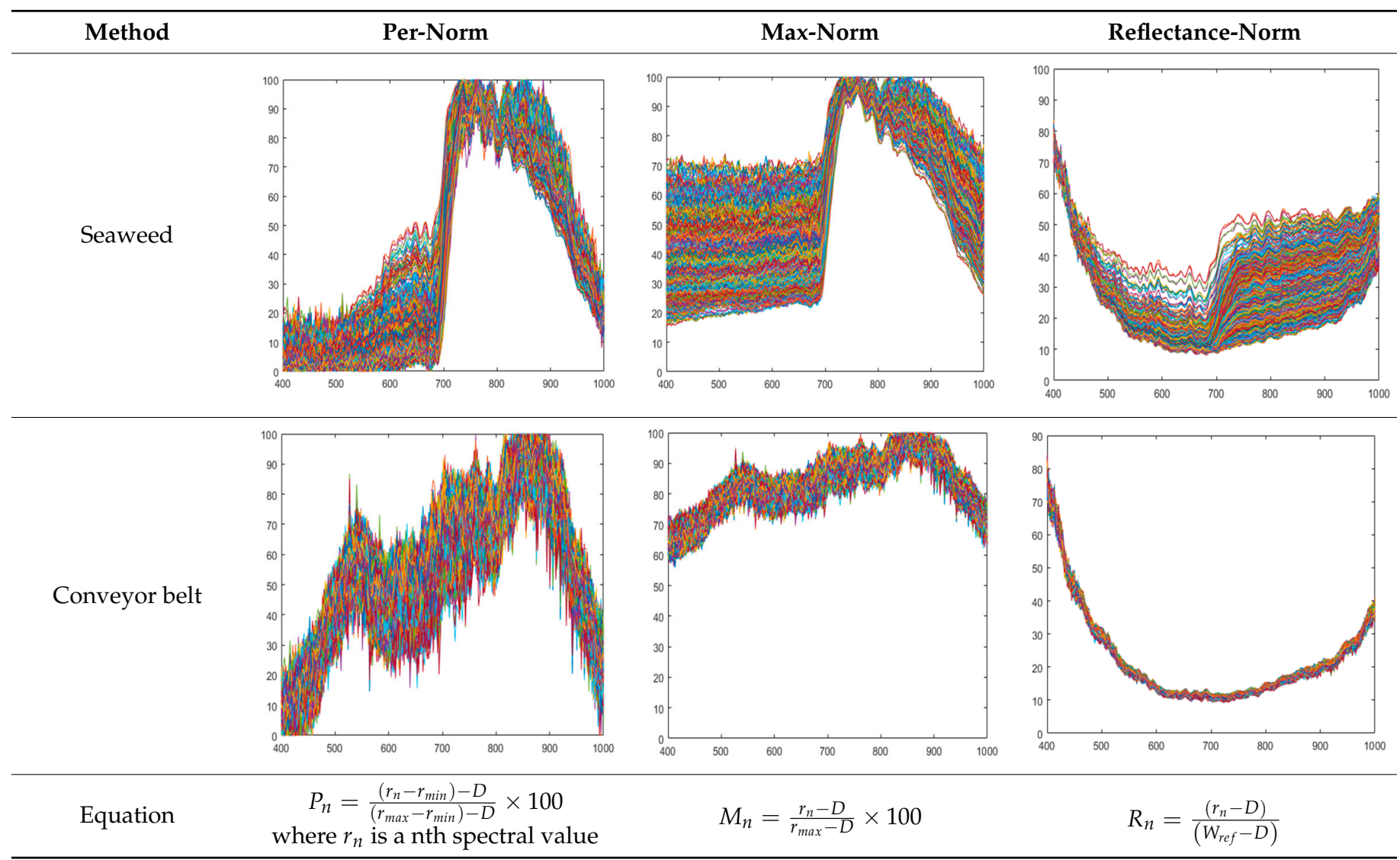

\subsubsection{Dimensionality Reduction}

Dimensionality reduction is essential in order to process massive amounts of hyperspectral data in real-time $[24,25]$. There are several methods of dimensionality reduction, but it is important to maintain the characteristics as much as possible in order to be prepared for unknown foreign objects. In this study, we used the spectral bining function included in the camera. This is a simplified function that acquires only $1 / \mathrm{n}$ of the data. For example, if use $1 / 2$ binning, skip the data one by one and acquire it. Even if the camera does not have this function, it can be easily implemented. When applying $1 / 2$ binning, the amount of data to be processed is reduced by $34,406,400$ bytes per line, and when applying $1 / 4$ binning, 51,609,600 bytes per line is reduced. In other words, it significantly reduces calculation overhead and enables real-time inspection. Figure $4 \mathrm{a}-\mathrm{c}$ shows the raw data, shows $1 / 2$ binning, and $1 / 4$ binning results, respectively. The application of $1 / 4$ binning loses some of the spectral characteristics for the peak. Therefore, we used $1 / 2$ binning and confirmed that it achieves real-time data processing.

\subsection{Proposed Algorithm}

The proposed detection algorithm proceeds in 2-stages. Firstly, the conveyor and seaweed are clearly distinguished by subtraction method. Secondly, detailed foreign object inspection is carried out precisely by standardization inspection. All the operations used in the algorithm are simplified and burdenless calculations such as subtraction, division, and one-by-one matching, so it can even run high-speed inspections $(30 \mathrm{~cm} / \mathrm{s})$ in real-time. 


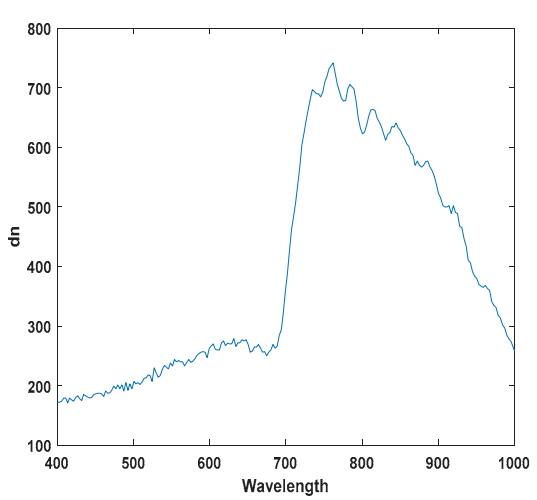

(a)

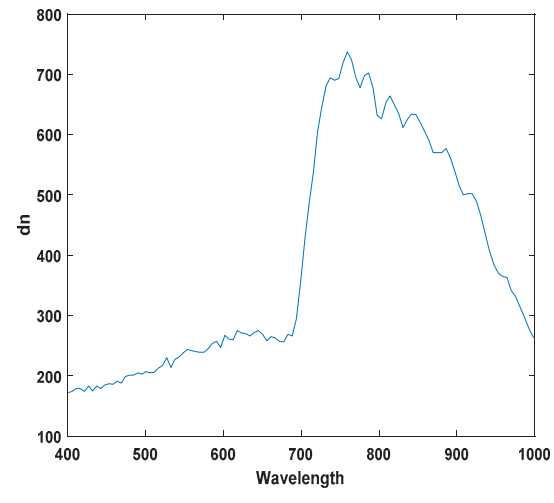

(b)

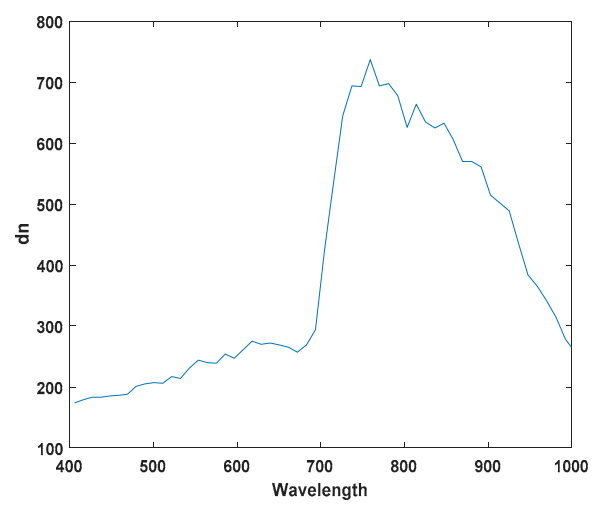

(c)

Figure 4. Spectral characteristics for each step of spectral binning (a) Full bands (224 bands) (b) $1 / 2$ binning applied (112 bands), (c) 1/4 binning applied (56 bands).

\subsubsection{Distinguishment between Seaweed and Conveyor Belt}

In the first stage, the subtraction method uses the difference in values between two different wavelengths in each wavelength range (visible range $(400-750 \mathrm{~nm})$ and nearinfrared range $(750-1000 \mathrm{~nm})$. The subtraction value allows two different subtraction images to be obtained and it also clearly distinguishes seaweed (target) and conveyor belt (background) through threshold setting. Even if the target object changes, you can change the band combination and threshold settings to easily distinguish the target from the background. The average of the values obtained from Table 3 (Per-Norm applied data) was used as reference data for seaweed and conveyor belt. Firstly, the wavelength value with the largest difference of subtraction value between seaweed and conveyor belt shall be selected. We calculated all cases in an empirical way to find the best band combination with the largest difference between the subtraction value of seaweed and conveyor belt. As a result, the 26th $(532.25 \mathrm{~nm})$ and 63rd $(731.79 \mathrm{~nm})$ bands $\left(P_{26}\right.$ and $\left.P_{63}\right)$ are selected in the visible range $\left(S_{V}\right)$, and the 68th $(759.14 \mathrm{~nm})$ and 93th $(897.28 \mathrm{~nm})$ bands $\left(P_{68}\right.$ and $\left.P_{93}\right)$ are selected in the near-infrared range $\left(S_{N}\right)$, respectively. When seaweed and conveyor belts can be clearly distinguished, there are two benefits to this operation. Firstly, it significantly reduces unnecessary calculations by ignoring the standardization inspection of the conveyor belt part (Figure 5 shows ignored zone). This is because foreign objects on the conveyor belt do not need to be detected. The ignored zone is about $20 \%$ of the total seaweed area, which can save a lot of computation. Secondly, it is possible to apply operations optimized for each of the seaweed pixels and conveyor belt pixels. In addition, accuracy also be improved by using 2-class classification instead of 3-class classification. Two types of subtraction images can be seen in Figure 6b,c.

$$
S_{V}=\mathrm{P}_{63}-\mathrm{P}_{26} S_{N}=\mathrm{P}_{93}-\mathrm{P}_{68}
$$

where $S_{V}$ is subtracted value of the visible range, and $S_{N}$ is subtracted value of the nearinfrared range.

\subsubsection{Detection of Foreign Object}

In the second stage, the standardization inspection is used for the detection of foreign objects and inspects after standardizing the pixels. The standard deviation for each seaweed and conveyor belt was calculated from the data in Table 3 (Per-Norm applied). The equation of standardization is shown in Equation (2). After standardizing pixels, the offset between the pixels is dramatically reduced.

$$
x_{n e w}=\frac{x-\mu}{\sigma}
$$


where $\mu$ is mean value of $x$, and $\sigma$ is standard deviation of $x$.

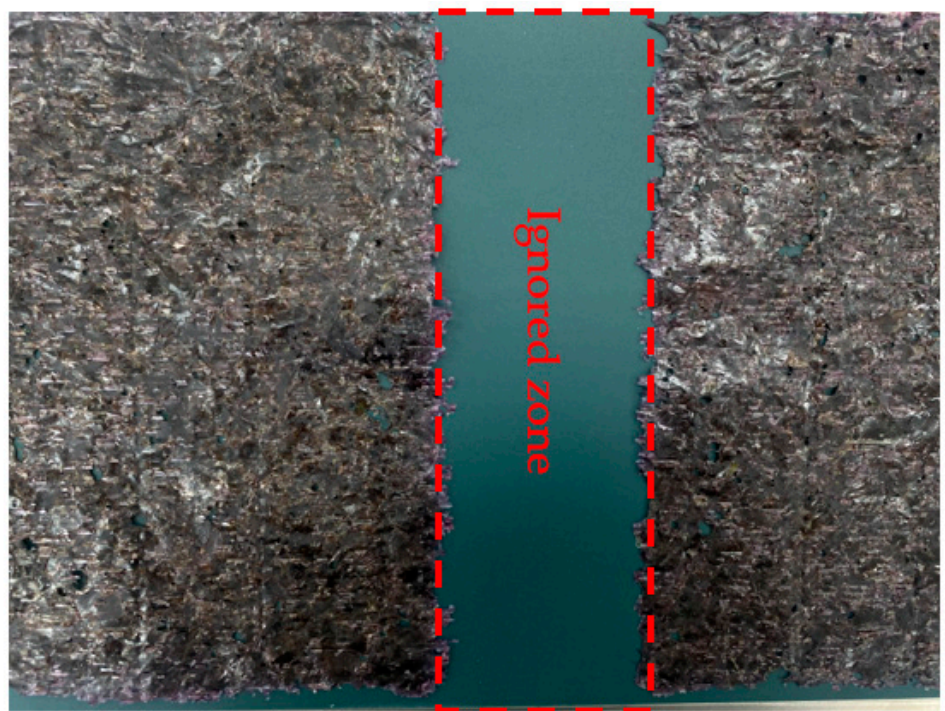

Figure 5. Example of an ignored zone.

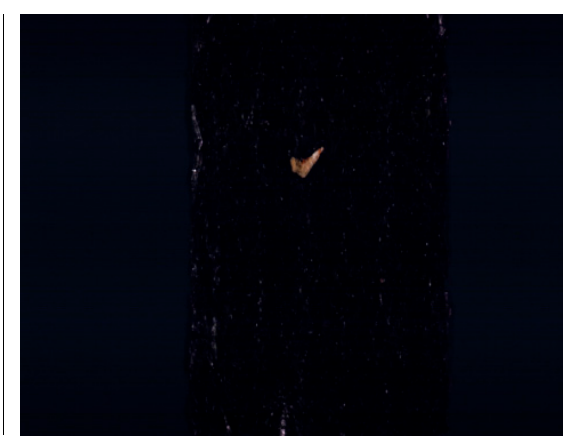

(a)

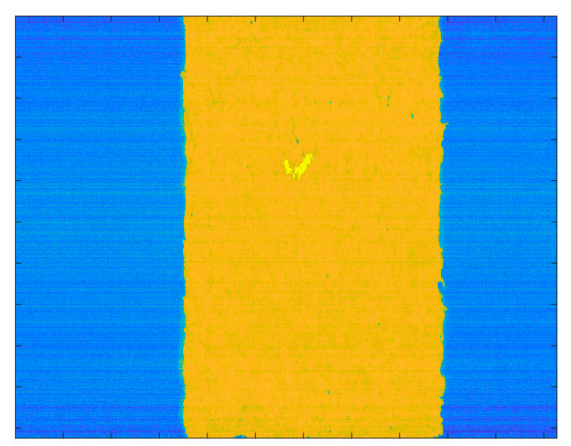

(b)

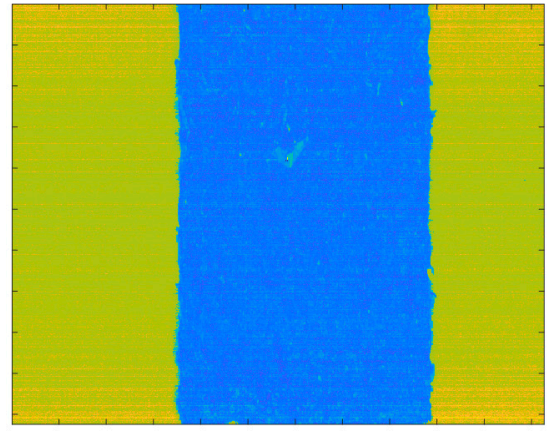

(c)

Figure 6. (a) Raw image, (b) Subtraction image in the visible range (c) Subtraction image in the near-infrared range.

\subsection{Multi-Class Support Vector Machine}

Multi-class Support Vector Machine (MCSVM) was adopted to compare the performance with the proposed algorithm. SVM [26] is a supervised learning model as one of the fields of machine learning, and is mainly used for classification and regression analysis. Basically, SVM is a binary classifier and a linear classifier, but it can be extended as a multi-class classifier and a nonlinear classifier. In order to perform nonlinear and multi-class classification, data must be mapped into a high-dimensional feature space. Kernel tricks are used to do this efficiently. In this experiment, we used the Spectral Angle Mapper (SAM) kernel-based SVM to test the algorithm. SAM calculates the angle in the n-dimensional space between the trained and test pixels. Classify through this angle. SAM kernel is widely used in hyperspectral imaging because it can quickly measure and classify the similarity of spectral angles [27-30]. The equation of SAM is shown in (3). We used the same input as the proposed algorithm as the input of SAM (Per-Norm and 1/2 binning applied).

$$
\theta(\mathrm{x}, \mathrm{y})=\cos ^{-1}\left(\frac{\sum_{i=1}^{n} x_{i} y_{i}}{\left(\sum_{i=1}^{n} x_{i}^{2}\right)^{\frac{1}{2}} \times\left(\sum_{i=1}^{n} y_{i}^{2}\right)^{\frac{1}{2}}}\right)
$$

where $x$ is spectral value of test pixel, $y$ is spectral value of trained pixel, and $n$ is the number of bands. 


\section{Results and Discussion}

\subsection{Detection Results of Proposed Algorithm}

In the experiment, pre-obtained hyperspectral images were used to test the performance of the proposed algorithm. This is the reason for measuring quantitative values in the same environment. Before applying the algorithm, the hyperspectral image was calibrated through Per-Norm, and the $1 / 2$ binning function was used to reduce the number of bands in half (112 bands).

\subsubsection{Subtraction Method}

The subtraction image in Figure $6 b, c$ shows that seaweed and conveyor belts are clearly classified. Threshold settings in the visible light range, the seaweed has a subtraction value of 60 to 80 and a conveyor belt has a subtraction value of 20 to 45, respectively. And in the near-infrared range, seaweed has a subtraction value of -30 to -5 and a conveyor belt has a subtraction value of 5 to 25, respectively. According to this result, thresholds were used to distinguish seaweed and conveyor belts. In addition, the remaining values are classified as foreign objects. It can be seen that the shrimp shell, which is relatively easy to distinguish, can be detected through the subtracted image in the visible light range. Seaweed and conveyor belts that are well classified in both the visible and near-infrared ranges, follow the results of the visible light range. In the case of foreign objects, it is classified as a foreign object if it is found in either of both ranges. Figure 7 shows the classification results through the subtraction method.

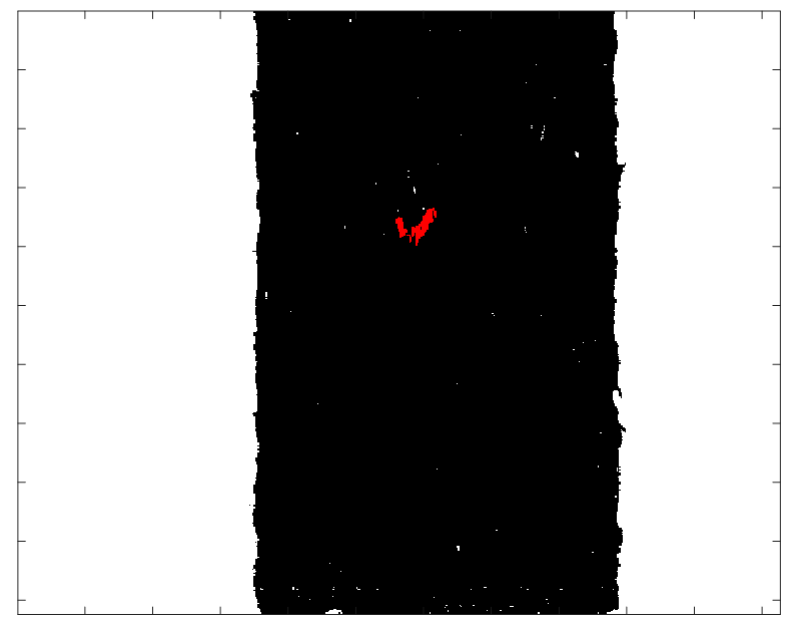

Figure 7. Classification result of the subtraction method.

\subsubsection{Standardization Inspection}

The results of applying standardization to reference data (Table 3 Per-Norm applied data) are shown in Figure 8. The limit range can be specified by giving margins to the maximum and minimum values of each band. In this experiment, a margin of 3 was given. If a value is outside the limit range, the pixel is considered a foreign object. Reducing the margins can increase sensitivity while increasing margins can reduce sensitivity. As an example, to test the performance of the standardization inspection [31], a piece of black plastic bag was used as a foreign object. Figure 9a raw hyperspectral image shows that it is difficult to detect a piece of black plastic bag even with the naked eyes. Figure $9 \mathrm{~b}$ shows that plastic bag detection failed by subtraction method. Some pixels of black plastic bags were classified as seaweed and some as conveyor belts. After applying standardization, the pixel values of the black plastic bag are shown in Figure 10. In seaweed pixels, values out of range occurred near $530-700 \mathrm{~nm}$ and $880-100 \mathrm{~nm}$ wavelengths, and in conveyor belts pixels, values out of range occurred near $400 \mathrm{~nm}$ and $780 \mathrm{~nm}$ wavelengths. Finally, the successful detection of a piece of black plastic bag is shown in Figure 8, and the overall process of the proposed algorithm is shown in Figure 11. 


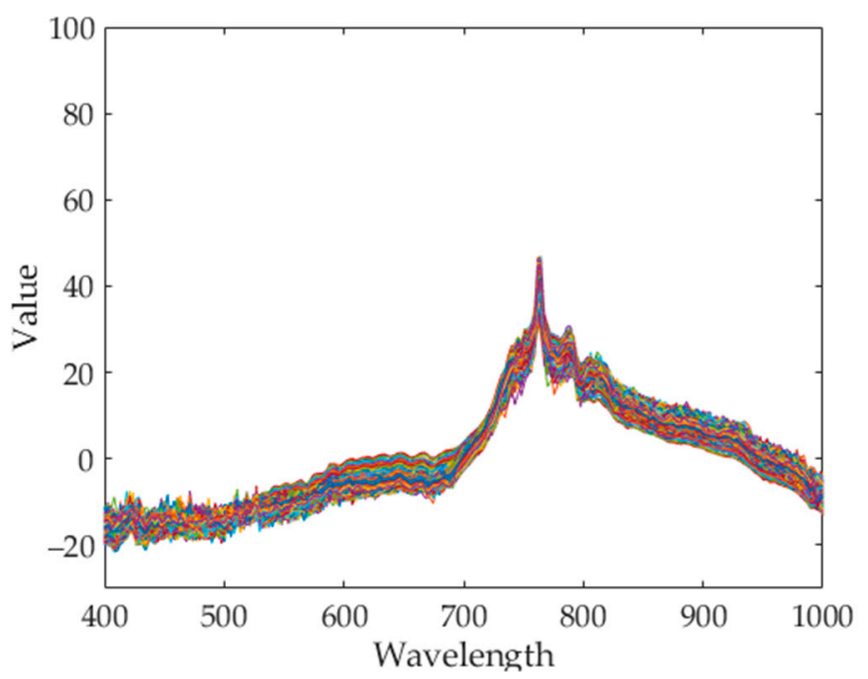

(a)

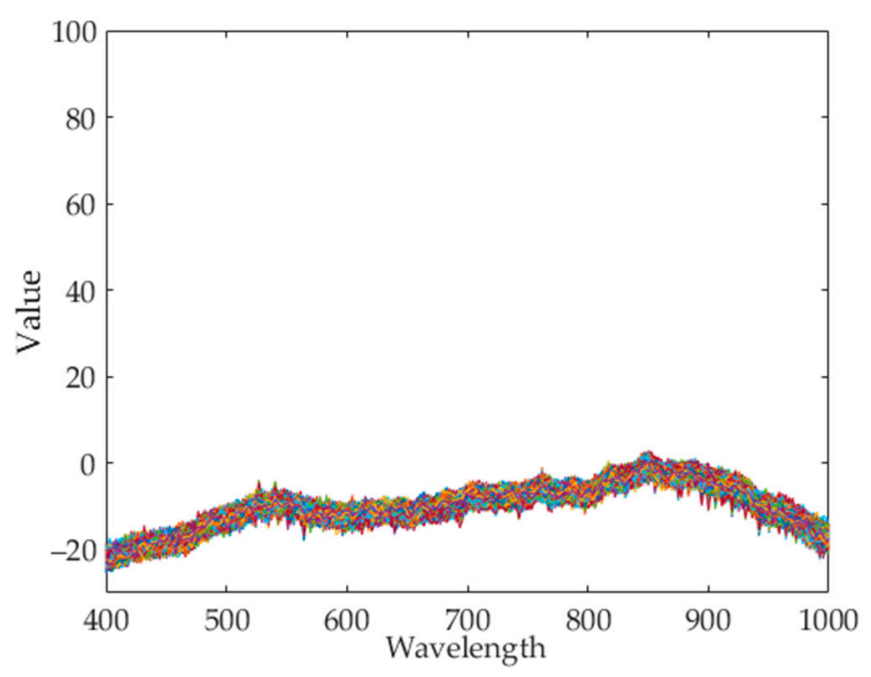

(b)

Figure 8. Result of standardization (a) Seaweed (b) Conveyor belt.

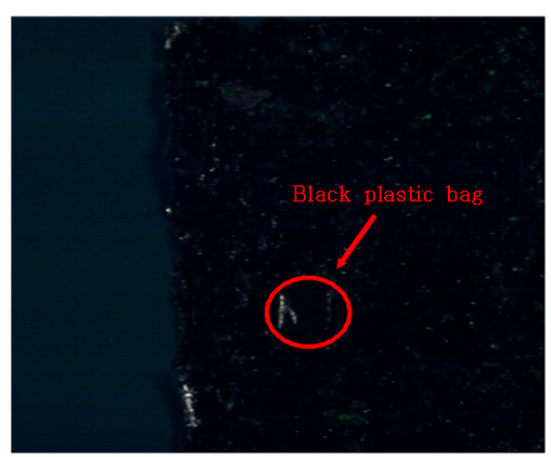

(a)

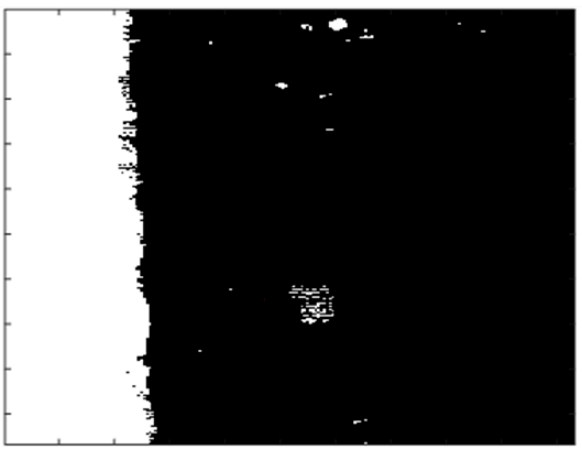

(b)

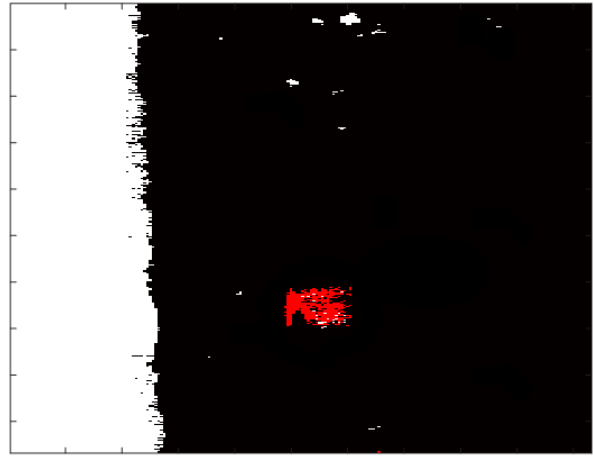

(c)

Figure 9. Results of each inspection step. (a) Raw hyperspectral image, (b) After applying the subtraction method (c) After applying the standardization inspection.

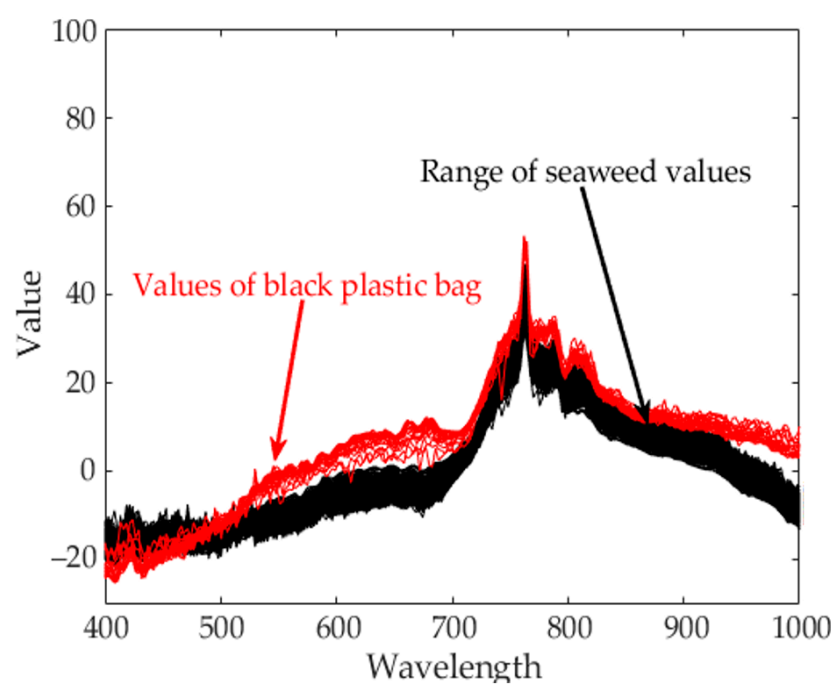

(a)

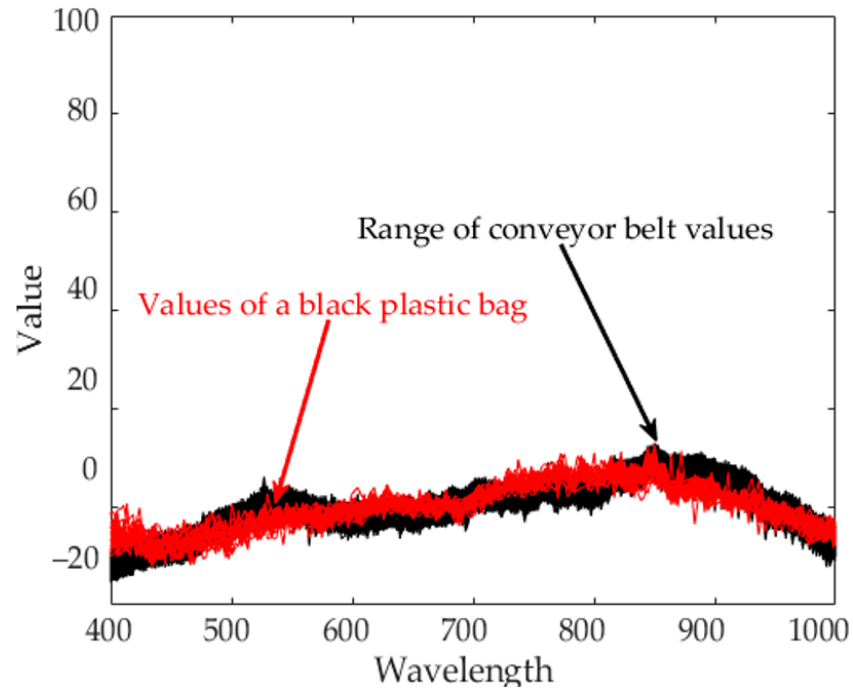

(b)

Figure 10. The range of the reference data and the value of the black plastic bag obtained after standardizing. (a) About seaweed pixels (b) About conveyor belt pixels. 


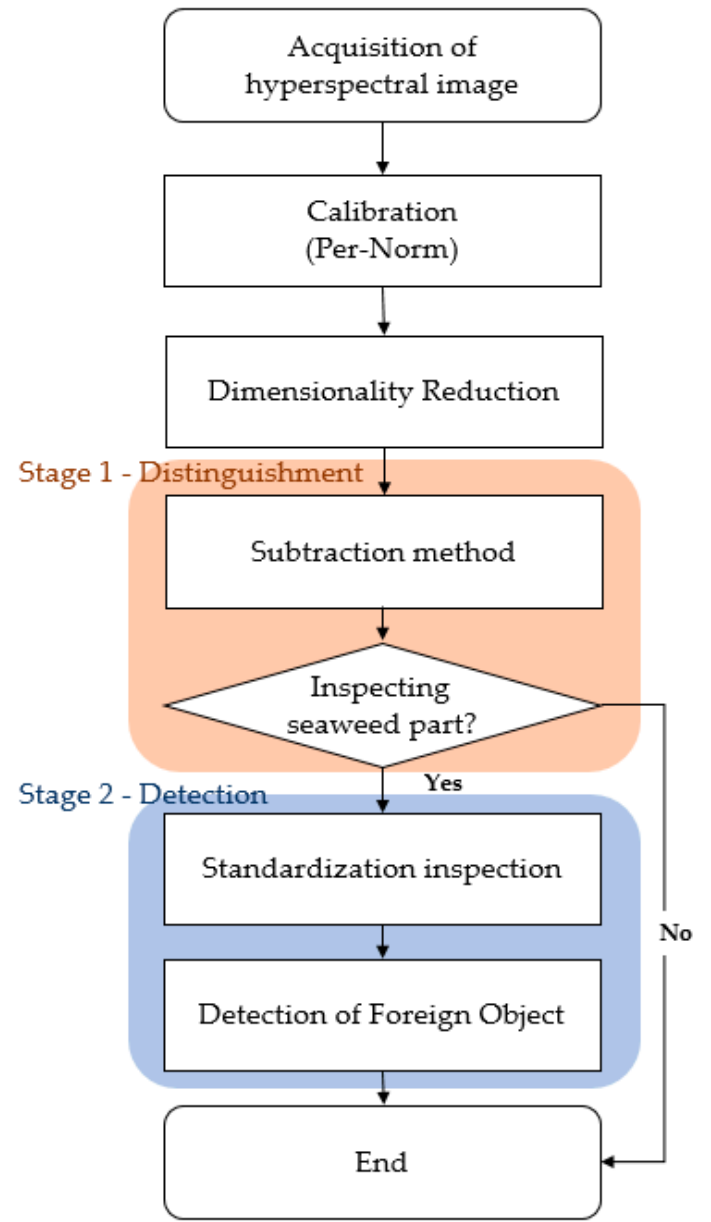

Figure 11. Flow chart of overall process.

\subsection{Detection Results of MCSVM}

The multi-class support vector machine (MCSVM) used in the experiment is a threeclass (Seaweed, conveyor belt, and foreign objects) classifier using a 112-dimensional SAM kernel. As an example, to test the performance of MCSVM, we used the same hyperspectral image used in the proposed algorithm. Both shrimp shells and black plastic bags were successfully detected. However, it can be seen from Figure 12a that many FP (False-Positive) pixels have occurred.

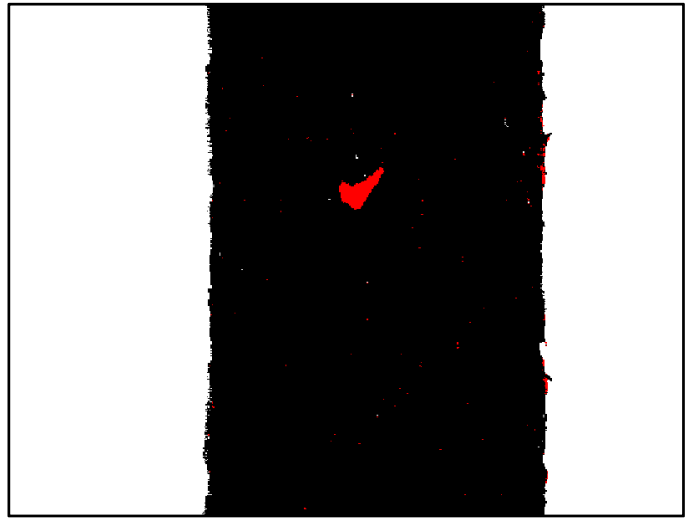

(a)

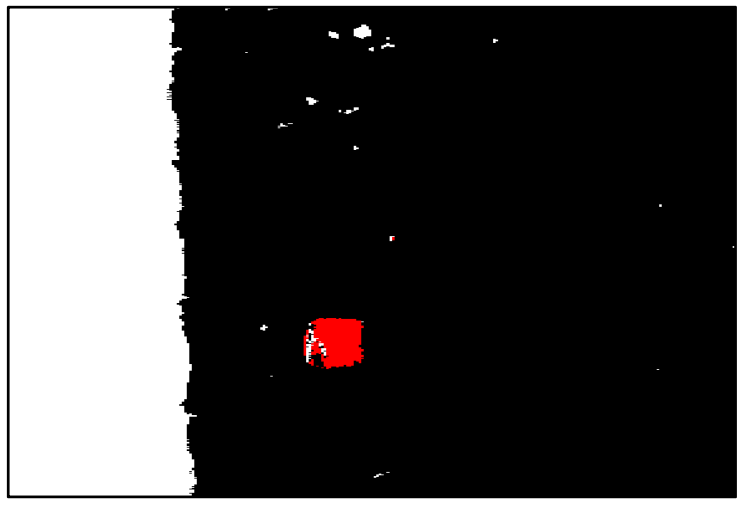

(b)

Figure 12. Classification result of the MCSVM. (a) Shrimp Shell (b) Black plastic bag. 


\subsection{Detection Performance of Each Algorithm}

To measure the quantitative performance of the proposed algorithm, 60 normal seaweed samples and 60 seaweed samples containing foreign objects were used, and 2 kinds of seaweeds were used. If there are pixels classified as foreign objects at least 1 pixel in the position of foreign objects, the detection is considered successful (True-Negative (TN)). In contrast, if at least 1 foreign object pixel is found in normal seaweed, it is considered misclassification (FN). The MCSVM has excellent foreign object classification performance. However, a number of FN have occurred. If about $30 \%$ of normal seaweed is rejected, it will have a fatal effect on productivity. In addition, the processing speed per line is about $60 \mathrm{~ms}$, so real-time inspection cannot be performed. The proposed algorithm also shows prominent detection performance. FN has not occurred, and the processing speed per line is about $3 \mathrm{~ms}$, which can operate in real-time even at the maximum frame rate ( $330 \mathrm{fps}$ ). Considering the various performances, the proposed algorithm is suitable for applications in real manufacturing sites. The detection performance of each algorithm can be seen in Table 4.

Table 4. Detection performance of each algorithm.

\begin{tabular}{ccccccccc}
\hline \multirow{2}{*}{ Method } & \multicolumn{4}{c}{ Detection Result } & \multicolumn{3}{c}{ Performance } & Processing Time/Line \\
\cline { 2 - 7 } & TP & FN & FP & TN & Recall & Precision & Accuracy & $3 \mathrm{~ms}$ \\
\hline Proposed algorithm & 60 & 0 & 6 & 54 & 1 & 0.91 & 0.95 & $60 \mathrm{~ms}$ \\
\hline MCSVM & 39 & 21 & 4 & 56 & 0.65 & 0.91 & 0.79 & \\
\hline
\end{tabular}

\subsection{Foreign Object Detection Platform}

The foreign object detection platform was implemented using the proposed algorithm to verify its applicability in the real manufacturing site. It was implemented in the Visual studio 2017 MFC environment and used the $\mathrm{C}++$ language. After classifying the pixels using the proposed algorithm, the display color is converted (seaweed-black, conveyor belt-white, foreign object-red) to increase the visibility of the classified results. Through this platform, we confirmed that the proposed algorithm works well at maximum fps. The platform can be seen in Figure 13.

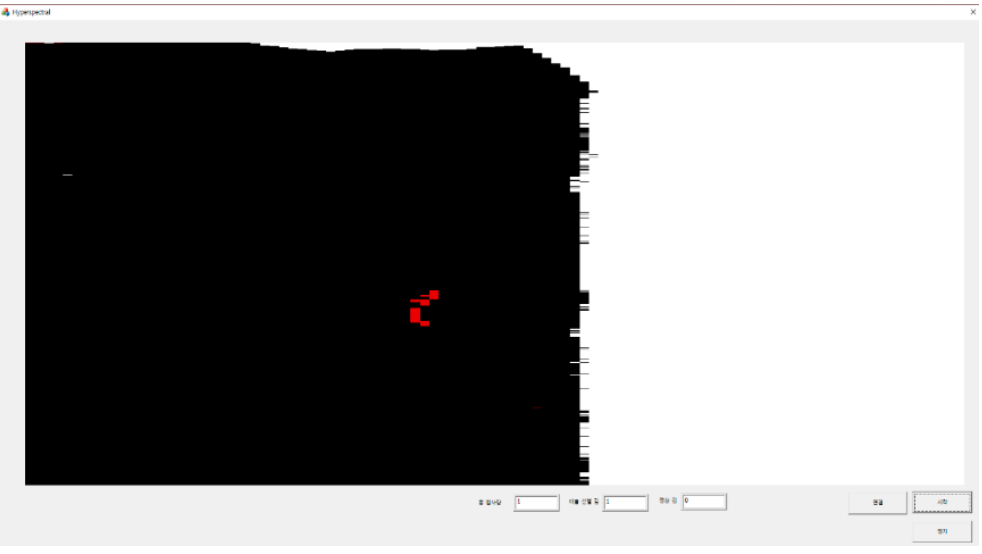

Figure 13. Foreign object detection platform.

\section{Conclusions}

The purpose of this study is to confirm the possibility of rapid and accurate foreign object detection using hyperspectral imaging technique. The proposed algorithm uses a simplified operations and dimensionality reduction for real-time inspection. In addition, efficiency and accuracy have been improved through a 2-stage detection method. In the first stage, the subtraction method distinguishes seaweed (target image) and conveyor belts (background image) very quickly and accurately by extracting the features of each 
of the visible and near-infrared ranges. In the visible range, the wavelength of $532.25 \mathrm{~nm}$ (26th band) and $731.79 \mathrm{~nm}$ (63rd band) were selected, and in the near-infrared range, the wavelength of $759.14 \mathrm{~nm}$ (68th band) and $897.28 \mathrm{~nm}$ (93rd band) were selected. At this stage, foreign objects that are relatively easy to detect can also be detected. Through this, unnecessary operations can be eliminated by excluding the conveyor belt part and foreign object pixels from the next inspection stage. In the second stage, the standardization inspection more carefully detects foreign objects which are difficult to detect by comparing the reference data by one-by-one matching. As a result of the experiment, the detection accuracy of proposed algorithm was $95 \%$, and the recall rate reached $100 \%$. The MCSVM used to compare the performance showed excellent foreign object detection performance, but a number of FN occurred. Additionally, MCSVM cannot perform rapid inspection in real-time, but the proposed algorithm can perform a real-time inspection even at maximum $\mathrm{fps}$, which has been revealed through platform implementation. Since the proposed algorithm easily compares and classifies the hyperspectral images of arbitrary foreign object and food, we expect that our work will be helpful in securing the safety of various food groups.

For future work, we plan to confirm the possibility of faster inspection through improved camera specifications. Currently, faster inspections are not possible due to camera specifications, but if the camera specifications are improved (higher maximum FPS), faster inspection is possible using higher dimensionality reduction ( $1 / 4$ binning). Rapid inspection technique using hyperspectral image can be applied not only in food products but also in other industries. Finally, we plan to explore the applicability of the proposed algorithm to various manufacturing industries.

Author Contributions: Conceptualization and methodology, D.-H.K. and Y.-D.K.; data curation, D.-H.K., G.-J.S., M.-K.P.; experiment, D.-H.K. and G.-J.S.; validation, D.-H.K., M.-K.P. and Y.-D.K.; resources, M.-K.P.; software implementation, D.-H.K. and G.-J.S.; writing, D.-H.K.; review and editing, Y.-D.K. and project administration, Y.-D.K. All authors have read and agreed to the published version of the manuscript.

Funding: This research was supported by a grant (no.19163MFDS521) from Ministry of Food and Drug Safety of Korea. And this work was also supported by DGIST research 373 grant (no.21-IT-02).

Institutional Review Board Statement: Not applicable.

Informed Consent Statement: Not applicable.

Conflicts of Interest: The authors declare no conflict of interest.

\section{References}

1. Wall Street Journal. Available online: https://www.wsj.com/articles/seaweed-fans-flock-to-expo-in-wando-south-korea-1410748201 (accessed on 17 May 2021).

2. Liu, B.; Zhou, W. The research of metal detectors using in food industry. In Proceedings of the 2011 International Conference on Electronics and Optoelectronics, Dalian, China, 29-31 July 2011; pp. 43-45. [CrossRef]

3. Kwon, J.-S.; Lee, J.-M.; Kim, W.-Y. Real-time detection of foreign objects using X-ray imaging for dry food manufacturing line. In Proceedings of the 2008 IEEE International Symposium on Consumer Electronics, Vilamoura, Portugal, 14-16 April 2008; pp. 1-4. [CrossRef]

4. Kuzy, J.; Li, C. A Pulsed Thermographic Imaging System for Detection and Identification of Cotton Foreign Matter. Sensors 2017, 17, 518. [CrossRef] [PubMed]

5. Shephard, D.J.; Tait, P.D.F.; King, R.J. Foreign object detection using radar. IEEE Aerosp. Conf. Proc. 2000, 6, 43-48. [CrossRef]

6. Lim, J.; Lee, A.; Kang, J.; Seo, Y.; Kim, B.; Kim, G.; Kim, S.M. Non-Destructive Detection of Bone Fragments Embedded in Meat Using Hyperspectral Reflectance Imaging Technique. Sensors 2020, 20, 4038. [CrossRef] [PubMed]

7. Weng, S.; Yu, S.; Guo, B.; Tang, P.; Liang, D. Non-Destructive Detection of Strawberry Quality Using Multi-Features of Hyperspectral Imaging and Multivariate Methods. Sensors 2020, 20, 3074. [CrossRef] [PubMed]

8. Yang, B.; Gao, Y.; Yan, Q.; Qi, L.; Zhu, Y.; Wang, B. Estimation Method of Soluble Solid Content in Peach Based on Deep Features of Hyperspectral Imagery. Sensors 2020, 20, 5021. [CrossRef] [PubMed]

9. Liu, Y.; Pu, H.; Sun, D.-W. Hyperspectral imaging technique for evaluating food quality and safety during various processes: A review of recent applications. Trends Food Sci. Technol. 2017, 69, 25-35. [CrossRef] 
10. Kim, M.; Chen, Y.; Mehl, P. Hyperspectral Reflectance and Fluorescence Imaging System for Food Quality and Safety. Trans. ASAE 2001, 44, 721-729. [CrossRef]

11. Manolakis, D.; Shaw, G. Detection algorithms for hyperspectral imaging applications. IEEE Signal Process. Mag. 2002, 19, 29-43. [CrossRef]

12. Landgrebe, D. Hyperspectral Image Data Analysis. IEEE Signal Process. Mag. 2002, 19, 17-28. [CrossRef]

13. Stein, D.; Beaven, S.; Hoff, L.; Winter, E.; Schaum, A.; Stocker, A. Anomaly Detection from Hyperspectral Imagery. IEEE Signal Process. Mag. 2002, 19, 19-58. [CrossRef]

14. Ren, S.; He, K.; Girshick, R.; Sun, J. Faster R-CNN: Towards Real-Time Object Detection with Region Proposal Networks. IEEE Trans. Pattern Anal. Mach. Intell. 2017, 39, 1137-1149. [CrossRef]

15. Bochkovskiy, A.; Wang, C.Y.; Liao, H.-Y.M. YOLOv4: Optimal Speed and Accuracy of Object Detection. arXiv 2020, arXiv:2004.10934.

16. Ronneberger, O.; Fischer, P.; Brox, T. U-Net: Convolutional Networks for Biomedical Image Segmentation. LNCS 2015, 9351, 234-241. [CrossRef]

17. Rong, D.; Xie, L.; Ying, Y. Computer vision detection of foreign objects in walnuts using deep learning. Comput. Electron. Agric. 2019, 162, 1001-1010. [CrossRef]

18. Hsieh, T.-H.; Kiang, J.-F. Comparison of CNN Algorithms on Hyperspectral Image Classification in Agricultural Lands. Sensors 2020, 20, 1734. [CrossRef] [PubMed]

19. Al-Sarayreh, M.; Reis, M.M.; Yan, W.Q.; Klette, R. A Sequential CNN Approach for Foreign Object Detection in Hyperspectral Images. In Proceedings of the 18th International Conference on Computer Analysis of Images and Patterns, Salerno, Italy, 3-5 September 2019; Springer: Berlin/Heidelberg, Germany, 2019; pp. 271-283. [CrossRef]

20. Specim Fx10. Available online: https://www.specim.fi/products/specim-fx10/ (accessed on 20 May 2021).

21. Lawrence, K.; Park, B.; Windham, W.; Mao, C. Calibration of a pushbroom hyperspectral imaging system for agricultural inspection. Trans. ASAE 2003, 46, 513-521. [CrossRef]

22. X-rite ColorChecker. Available online: https://www.xrite.com/categories/calibration-profiling/colorchecker-white-balance (accessed on 25 May 2021).

23. Kim, H.; Kim, S. Per-Norm Based Automotive Headlamp Detection in Hyperspectral Image for Visible Light Communication. J. Comput. Electr. Eng. 2016, 8, 12-21. [CrossRef]

24. Jihan, K.; Rafic, Y. Dimensionality reduction on hyperspectral images: A comparative review based on artificial datas. In Proceedings of the 2011 4th International Congress on Image and Signal Processing, Shanghai, China, 15-17 October 2011; pp. 1875-1883. [CrossRef]

25. Caicedo, J.P.R.; Verrelst, J.; Munoz, J.; Camps-Valls, G.; Moreno, J. Hyperspectral dimensionality reduction for biophysical variable statistical retrieval. ISPRS J. Photogramm. Remote Sens. 2017, 132, 88-101. [CrossRef]

26. Hearst, M.; Dumais, S.; Osuna, E.; Platt, J.; Scholkopf, B. Support vector machines. IEEE Intell. Syst. Appl. 1998, 13, 18-28. [CrossRef]

27. Sohn, Y.; Rebello, S. Supervised and Unsupervised Spectral Angle Classifiers. Photogramm. Eng. Remote Sens. 2002, 68, 1271-1280.

28. Liu, X.; Yang, C. A Kernel Spectral Angle Mapper algorithm for remote sensing image classification. In Proceedings of the 2013 6th International Congress on Image and Signal Processing (CISP), Hangzhou, China, 16-18 December 2013; pp. 814-818. [CrossRef]

29. Fauvel, M.; Chanussot, J.; Benediktsson, J. Evaluation of Kernels for Multiclass Classification of Hyperspectral Remote Sensing Data. In Proceedings of the 2006 IEEE International Conference on Acoustics Speech and Signal Processing Proceedings, Toulouse, France, 14-19 May 2006. [CrossRef]

30. Kim, H.; Kwon, S.; Kim, S. Hyperspectral Image-Based Night-Time Vehicle Light Detection Using Spectral Normalization and Distance Mapper for Intelligent Headlight Control. Sensors 2016, 16, 1058. [CrossRef] [PubMed]

31. Femenias, A.; Gatius, F.; Ramos, A.J.; Sanchis, V.; Marín, S. Standardisation of near infrared hyperspectral imaging for quantification and classification of DON contaminated wheat samples. Food Control 2020, 111, 107074. [CrossRef] 